\title{
Pioglitazone improves the cardio-ankle vascular index in patients with type 2 diabetes mellitus treated with metformin
}

This article was published in the following Dove Press journal:

Diabetes, Metabolic Syndrome and Obesity: Targets and Therapy

17 July 2014

Number of times this article has been viewed

\author{
Masahiro Ohira' \\ Takashi Yamaguchi' \\ Atsuhito Saiki' \\ Noriko Ban' \\ Hidetoshi Kawana' \\ Ayako Nagumo' \\ Takeyoshi Murano 2 \\ Kohji Shirai ${ }^{3}$ \\ Ichiro Tatsuno' \\ 'Center for Diabetes, Endocrinology \\ and Metabolism, ${ }^{2}$ Department \\ of Clinical Laboratory Medicine, \\ ${ }^{3}$ Department of Vascular Function, \\ Sakura Hospital, Toho University \\ Medical Center, Chiba, Japan
}

Background: Type 2 diabetes is known to be associated with elevated cardiovascular mortality. Pioglitazone improves blood pressure (BP) and pulse wave velocity (PWV), which is an arterial stiffness parameter. Arterial stiffness is closely associated with cardiovascular disease. However, PWV is correlated with BP. The cardio-ankle vascular index (CAVI) reflects arterial stiffness independent of BP. Pioglitazone improves PWV but reduces blood pressure. The aim of this study was to re-evaluate the effect of pioglitazone on arterial stiffness with CAVI.

Methods: Sixty patients with type 2 diabetes mellitus and already on $500 \mathrm{mg} /$ day of metformin received add-on therapy of pioglitazone $15 \mathrm{mg}$ /day or glimepiride $1 \mathrm{mg} /$ day for 6 months, during which time changes in their metabolic parameters and CAVI were observed.

Results: After 6 months of treatment, both pioglitazone $(n=30)$ and glimepiride $(n=30)$ improved fasting blood glucose and glycated hemoglobin. The changes in fasting blood glucose and glycated hemoglobin between the two groups were greater in the pioglitazone group. Systolic and diastolic BP was decreased in both groups, with no significant between-group differences. Only pioglitazone increased serum adiponectin levels, and the change in adiponectin between the pioglitazone and glimepiride groups was significantly different. CAVI was decreased significantly by pioglitazone but remained unchanged after treatment with glimepiride. The change in CAVI between the two groups was significantly different.

Conclusion: These results suggest that pioglitazone improves CAVI, a BP-independent arterial stiffness parameter, in patients with type 2 diabetes mellitus treated with metformin.

Keywords: biguanide, thiazolidinediones, sulfonylurea, blood pressure, cardiovascular disease, arterial stiffness

\section{Introduction}

Cardiovascular disease is a major cause of death in patients with type 2 diabetes. ${ }^{1}$ Intensive glycemic control reduces microvascular complications; however, the effect on cardiovascular disease is rather limited. ${ }^{2}$ Control of blood glucose levels is one of the principal purposes of treating diabetes; however, it has been difficult to demonstrate a beneficial effect of well controlled blood glucose on the macrovascular complications of diabetes. ${ }^{3,4}$ The United Kingdom Prospective Diabetes Study (UKPDS) demonstrated that the risk of cardiovascular morbidity and mortality was reduced in patients with type 2 diabetes receiving intensive glucose control using metformin. ${ }^{5}$ Metformin improves insulin sensitivity and is thus important in the management of traditional cardiovascular risk factors, such as a high glycosylated hemoglobin $\left(\mathrm{HbA}_{1 \mathrm{c}}\right)$ level, hypertension, dyslipidemia, and central obesity. All of these traditional cardiovascular risk factors are associated with insulin resistance. ${ }^{6}$ 
Some nontraditional cardiovascular risk factors have been reported. Oxidized low-density lipoprotein (LDL), which represents one type of modified LDL, is considered to be important in the progression of atherosclerosis. ${ }^{7}$ Adiponectin is a well known adipocytokine. The serum adiponectin level reflects the degree of insulin resistance. ${ }^{8}$ The lipoprotein lipase mass level in preheparin serum (preheparin LPL mass) is associated with metabolic syndrome. ${ }^{9}$

However, metformin monotherapy cannot always control hyperglycemia, and other hypoglycemic agents sometimes have to be coadministered. Sulfonylureas have been used as insulin secretagogues and are one of the most commonly used classes of antidiabetic agents. Thiazolidinediones (TZDs) are antidiabetic agents that reduce blood glucose to increase insulin sensitivity in peripheral tissues..$^{10}$ Antiatherogenic effects have been reported for both sulfonylureas and TZDs. ${ }^{11,12}$ TZDs have beneficial effects on lipid metabolism, such as enlarging LDL particle size in patients with type 2 diabetes. ${ }^{13}$ In addition, TDZ improves pulse wave velocity (PWV) in Japanese diabetic patients. ${ }^{14}$

Brachial-ankle PWV (baPWV) has been used to evaluate arterial stiffness and atherosclerosis. An arterial stiffness parameter, cardio-ankle vascular index (CAVI), was developed as a marker of arteriosclerosis involving the aorta, femoral artery, and tibial artery. ${ }^{15} \mathrm{CAVI}$ is measured from an electrocardiogram, phonocardiogram, brachial artery waveform, and ankle artery waveform and is adjusted for blood pressure (BP) based on the stiffness parameter $\beta .{ }^{16} \mathrm{CAVI}$ is independent of $\mathrm{BP}$ and has adequate reproducibility for clinical use, but baPWV is dependent on BP. ${ }^{15}$ Indeed, baPWV is decreased by metoprolol, a $\beta 1$-selective adrenergic receptor blocker with limited arterial effects. ${ }^{17}$ On the other hand, CAVI is not decreased by metoprolol. ${ }^{17}$ Both baPWV and CAVI are decreased by doxazosin, an $\alpha 1$-adrenergic receptor blocker with a strong vasodilating effect. ${ }^{17}$ These results also indicate that CAVI, not baPWV, reflects arterial stiffness independent of BP. Although arterial stiffness can be evaluated by measuring either baPWV or CAVI, CAVI is superior to baPWV as an index of arterial stiffness in patients who have undergone coronary angiography. ${ }^{16}$ Some reports indicate that CAVI is useful for detecting atherosclerotic disease ${ }^{15,18}$ and is superior to intima-media thickness for predicting coronary atherosclerosis. ${ }^{19} \mathrm{We}$ considered that we needed to re-evaluate the effect of TZDs on arterial stiffness with CAVI because pioglitazone decreases both BP and PWV, which is a blood pressure-dependent arterial stiffness parameter. ${ }^{14}$

In this study, we reinvestigated the effect of TZDs on arterial stiffness with CAVI. We hypothesized that TZDs improve arterial stiffness by improving not only glucose metabolism but also some nontraditional cardiovascular risk factors. We administered sulfonylureas or TZD as add-on therapy in type 2 diabetic patients receiving metformin and evaluated the effect of the combination on CAVI, a BPindependent arterial stiffness parameter and a surrogate marker of cardiovascular disease.

\section{Materials and methods}

The study protocol was prepared in accordance with the Declaration of Helsinki and was approved by the institutional review board of Sakura Hospital, Toho University Medical Center (approval number 2012-114). Before participation, we explained the purpose of the study to each subject, and consent was obtained for both participation in this study and for release of the study data.

\section{Study subjects}

A randomized open-label study was performed. Sixty patients with type 2 diabetes $\left(\mathrm{HbA}_{1 \mathrm{c}}>7.0 \%\right)$ were enrolled. At baseline, all subjects were treated solely with $500 \mathrm{mg}$ /day of metformin, which acted as an oral hypoglycemic agent. We divided the patients into two groups by simple randomization using a closed envelope. One group was administered pioglitazone (a TZD) at $15 \mathrm{mg} /$ day (pioglitazone group, $\mathrm{n}=30$ ), and the other group was administered glimepiride (a sulfonylurea) at $1 \mathrm{mg}$ /day (glimepiride group, $\mathrm{n}=30$ ). Table 1 shows the clinical characteristics of the subjects at baseline. All subjects were observed for 6 months after initiation of pioglitazone or glimepiride add-on therapy, and the following parameters were measured after 6 months: body weight, body mass index, fasting blood glucose, $\mathrm{HbA}_{1 \mathrm{c}}$, serum total cholesterol, triglycerides, high-density lipoprotein cholesterol, LDL cholesterol, preheparin LPL mass, adiponectin, and malondialdehyde-modified LDL levels, as well as LDL particle size and urinary albumin. Systolic BP, diastolic BP, and CAVI were also measured before and after 6 months. All patients maintained the same diet and exercise therapies and did not change medications during this study. A dietician provided nutritional guidance to all subjects on a monthly basis, analyzing meals and suggesting changes if necessary.

\section{Measurement of body weight and blood sampling}

Measurement of body weight and collection of blood were done in the morning after 12 hours of fasting. Serum was separated within one hour, and the sample was used to measure 
Table I Comparisons of baseline characteristics in the two groups

\begin{tabular}{|c|c|c|c|}
\hline & $\begin{array}{l}\text { Pioglitazone } \\
\text { group }\end{array}$ & $\begin{array}{l}\text { Glimepiride } \\
\text { group }\end{array}$ & $P$ value \\
\hline $\begin{array}{l}\text { No of subjects } \\
\text { (male/female) }\end{array}$ & $30(19 / 11)$ & $30(15 / 15)$ & NS \\
\hline Age (years) & $63.70 \pm 7.94$ & $62.23 \pm 12.26$ & NS \\
\hline BW (kg) & $61.84 \pm 16.96$ & $63.70 \pm 15.85$ & NS \\
\hline BMI $\left(\mathrm{kg} / \mathrm{m}^{2}\right)$ & $23.6 \mathrm{I} \pm 4.06$ & $24.49 \pm 4.54$ & NS \\
\hline $\mathrm{SBP}(\mathrm{mmHg})$ & $|46.87 \pm| 4.9 \mid$ & $|40.50 \pm| 4.84$ & NS \\
\hline $\mathrm{DBP}(\mathrm{mmHg})$ & $85.87 \pm 9.60$ & $85.17 \pm 11.28$ & NS \\
\hline FBG (mg/dL) & $221.23 \pm 80.68$ & $218.27 \pm 77.60$ & NS \\
\hline $\mathrm{HbA}_{\mathrm{lc}}(\%)$ & $8.54 \pm 1.06$ & $8.64 \pm 1.34$ & NS \\
\hline TC (mg/dL) & $199.30 \pm 36.97$ & $202.00 \pm 32.73$ & NS \\
\hline TG (mg/dL) & $177.67 \pm \mid 02.47$ & $163.63 \pm 89.52$ & NS \\
\hline HDL-C (mg/dL) & $49.60 \pm 12.43$ & $50.30 \pm 12.79$ & NS \\
\hline LDL-C (mg/dL) & $|17.90 \pm 32.8|$ & $|20.77 \pm 3| .32$ & NS \\
\hline $\begin{array}{l}\text { Preheparin LPL mass } \\
(\mathrm{ng} / \mathrm{mL})\end{array}$ & $50.21 \pm 17.53$ & $48.29 \pm 18.00$ & NS \\
\hline Adiponectin (ng/mL) & $7.32 \pm 5.48$ & $8.40 \pm 6.08$ & NS \\
\hline MDA-LDL (U/L) & $104.93 \pm 74.59$ & $96.10 \pm 27.58$ & NS \\
\hline Urinary albumin (mg/g Cr) & $386.8 I \pm 849.7 \mid$ & $|3| .50 \pm 28 \mid .65$ & NS \\
\hline LDL-Rm ratio & $0.3483 \pm 0.033$ & $0.3420 \pm 0.033$ & NS \\
\hline CAVI & $9.59 \pm 0.88$ & $9.32 \pm 144$ & NS \\
\hline
\end{tabular}

Note: Data are presented as mean \pm SD.

Abbreviations: BW, body weight; BMI, body mass index; SBP, systolic blood pressure; DBP, diastolic blood pressure; FBG, fasting blood glucose; $\mathrm{HbA}_{1 \mathrm{c}}$, glycosylated hemoglobin; TC, total cholesterol; TG, triglycerides; HDL-C, high density lipoprotein-cholesterol; LDL-C, low density lipoprotein-cholesterol; preheparin LPL mass, lipoprotein lipase in preheparin serum; MDA-LDL, malondialdehyde-modified low-density lypoprotein (LDL); LDL-Rm ratio, relative motility ratio of LDL on electrophoresis; CAVI, Cardio-Ankle Vascular Index; NS, not significant.

$\mathrm{HbA}_{1 \mathrm{c}}$, serum lipids, preheparin LPL mass, and adiponectin, and for analysis of serum lipoproteins by polyacrylamide gel disc electrophoresis.

\section{Measurement of $\mathrm{HbA}_{\mathrm{lc}}$ and plasma lipid concentrations}

To measure $\mathrm{HbA}_{1 \mathrm{c}}$, blood was collected in tubes containing ethylenediaminetetraacetic acid. The stable and unstable fractions of $\mathrm{HbA}_{1 \mathrm{c}}$ were measured by a high-pressure liquid chromatography method (Hi-Auto A1c analyzer system; Kyoto Daiichi Kagaku, Kyoto, Japan). Stable form data were used in the present analysis. $\mathrm{HbA}_{1 \mathrm{c}}$ was expressed as the value of the national glycohemoglobin standardization program (NGSP).

Plasma total cholesterol and triglyceride levels were measured enzymatically using kits from Nippon Shoji Co, Ltd (Osaka, Japan) and a 7150 analyzer (Hitachi, Ltd, Tokyo, Japan). Serum high-density lipoprotein cholesterol was measured using a selective inhibition assay (Daiichi Pure Chemicals Co, Ltd, Tokyo, Japan). ${ }^{20}$ Serum LDL cholesterol levels were calculated using the Friedewald formula.

\section{Serum preheparin LPL mass, adiponectin,} and malondialdehyde-modified LDL

Serum preheparin LPL mass was measured by a sandwich enzyme-linked immunosorbent assay (ELISA) using a specific monoclonal antibody against LPL (Daiichi Pure Chemicals Co, Ltd), as described by Kobayashi et al. ${ }^{21}$ The linearity and coefficient of variation are described in our previous report. ${ }^{22}$ The serum adiponectin level was measured using an adiponectin ELISA system (Otsuka Pharmaceutical Co, Tokushima, Japan). The ELISA for malondialdehydemodified LDL was based on the method reported by Kotani et $\mathrm{al}^{23}$ and is described in our previous report. ${ }^{22}$

\section{Urinary albumin}

The urinary albumin concentration was determined by turbidimetric immunoassay using a superior-microalbumin kit (LSI Medience Corporation, Tokyo, Japan). Urinary albumin was corrected for urinary creatinine concentration, which was measured by an enzymatic reaction.

\section{LDL particle size}

Serum lipoproteins were separated using polyacrylamide gel disc electrophoresis by the Lipo Phor ${ }^{\mathrm{TM}}$ system (Quantimetrix Corporation, Redondo Beach, CA, USA). ${ }^{24}$ LDL particle size was evaluated by examining the relative mobility of the peak of LDL fraction (LDL-Rm) determined from the densitometric pattern (Densitron 20-HR; Jokoh Co, Ltd., Tokyo, Japan). The LDL-Rm is significantly correlated with LDL particle size, and a decrease in the Rm ratio indicates an increase in LDL particle size in polyacrylamide gel disc electrophoresis. ${ }^{25}$

\section{CAVI and systolic and diastolic BP}

CAVI is obtained by measuring BP and PWV according to the following formula: $\mathrm{CAVI}=\mathrm{a}\{(2 \rho / \Delta \mathrm{P}) \times \ln (\mathrm{Ps} / \mathrm{Pd})$ $\left.\mathrm{PWV}^{2}\right\}+\mathrm{b}$, where Ps is systolic BP, Pd is diastolic BP, PWV is pulse wave velocity, $\Delta \mathrm{P}$ is $\mathrm{Ps}-\mathrm{Pd}, \rho$ is blood density, and $\mathrm{a}$ and $\mathrm{b}$ are constants. The details and measurement of CAVI are described in our previous reports. ${ }^{15,22}$ In the present study, CAVI was measured using a VaSera CAVI instrument (Fukuda Denshi Co, Ltd, Tokyo, Japan) as described previously. ${ }^{15}$ Systolic and diastolic BP was measured at the same time as CAVI measurement.

\section{Statistical analysis}

The data are expressed as the mean \pm standard deviation. Normal distribution was tested using the Shapiro-Wilk test. Normality was secured by logarithmic transformation, 
because some data were not normally distributed. Statistical analysis was performed using the Student's $t$-test and analysis of variance. All analyses were performed using JMP computer software version 9.0 (SAS Institute, Cary, NC, USA). $P$-values of $<0.05$ were considered to be statistically significant.

\section{Results}

The mean patient age was slightly higher in the pioglitazone group. The mean body weight and body mass index were slightly lower and urinary albumin was slightly higher in the pioglitazone group than in the glimepiride group. However, these differences were not significant. At baseline, the mean CAVI was slightly higher in the pioglitazone group than in the glimepiride group $(9.59 \pm 0.88$ versus $9.32 \pm 1.44)$, although the difference was not significant. Other parameters were almost identical in the two groups (Table 1).

Body weight and body mass index were increased in both groups, and the increase in body weight and body mass index was significantly higher in pioglitazone group than in the glimepiride group (Table 2). Both pioglitazone and glimepiride reduced fasting blood glucose and $\mathrm{HbA}_{1 \mathrm{c}}$ after 6 months of treatment. The change in fasting blood glucose was significant between the two groups. Serum adiponectin levels were increased only in the pioglitazone group. Comparison between the two groups revealed that

Table 2 Comparisons of the differentiation of each clinical parameter after 6 months in the two groups

\begin{tabular}{|c|c|c|c|}
\hline & \multicolumn{2}{|c|}{ Change from baseline } & \multirow[t]{2}{*}{$P$ value } \\
\hline & $\begin{array}{l}\text { Pioglitazone } \\
\text { group }\end{array}$ & $\begin{array}{l}\text { Glimepiride } \\
\text { group }\end{array}$ & \\
\hline$\Delta \mathrm{BW}(\mathrm{kg})$ & $+1.98 \pm 2.34$ & $+0.37 \pm 1.86$ & 0.0022 \\
\hline$\Delta \mathrm{BMI}\left(\mathrm{kg} / \mathrm{m}^{2}\right)$ & $+0.77 \pm 0.89$ & $+0.14 \pm 4.54$ & 0.0022 \\
\hline$\triangle \mathrm{SBP}(\mathrm{mmHg})$ & $-0.87 \pm 11.52$ & $-0.27 \pm 11.33$ & 0.8709 \\
\hline$\triangle \mathrm{DBP}(\mathrm{mmHg})$ & $-3.23 \pm 6.82$ & $-1.27 \pm 8.11$ & 0.2913 \\
\hline$\Delta \mathrm{FBG}(\mathrm{mg} / \mathrm{dL})$ & $-59.13 \pm 85.89$ & $-28.03 \pm 61.53$ & 0.0287 \\
\hline$\Delta \mathrm{HbA}_{\mathrm{lc}}(\%)$ & $-0.82 \pm 1.07$ & $-0.44 \pm 1.23$ & 0.1997 \\
\hline$\Delta \mathrm{TC}(\mathrm{mg} / \mathrm{dL})$ & $+2.97 \pm 30.28$ & $+2.47 \pm 18.12$ & 0.9553 \\
\hline$\Delta \mathrm{TG}(\mathrm{mg} / \mathrm{dL})$ & $-7.17 \pm 81.44$ & $-2.43 \pm 75.00$ & 0.7067 \\
\hline$\Delta \mathrm{HDL}-\mathrm{C}(\mathrm{mg} / \mathrm{dL})$ & $+3.30 \pm 6.19$ & $+0.00 \pm 6.42$ & 0.1148 \\
\hline$\Delta \mathrm{LDL}-\mathrm{C}(\mathrm{mg} / \mathrm{dL})$ & $-|.53 \pm 22.5|$ & $+4.30 \pm 12.97$ & 0.1568 \\
\hline $\begin{array}{l}\Delta \text { preheparin LPL } \\
\text { mass }(\mathrm{ng} / \mathrm{mL})\end{array}$ & $+13.00 \pm 15.43$ & $+7.60 \pm 13.77$ & 0.0563 \\
\hline$\Delta$ adiponectin (ng/mL) & $+6.43 \pm 4.89$ & $-0.09 \pm 3.04$ & $<0.0001$ \\
\hline$\Delta$ MDA-LDL (U/L) & $-0.08 \pm 0.27$ & $-0.43 \pm 31.61$ & 0.8445 \\
\hline $\begin{array}{l}\Delta \text { urinary albumin } \\
(\mathrm{mg} / \mathrm{g} \mathrm{Cr})\end{array}$ & $-20.58 \pm 233.23$ & $+51.69 \pm 351.71$ & 0.4578 \\
\hline$\Delta \mathrm{LDL}-\mathrm{Rm}$ ratio & $-0.0113 \pm 0.0300$ & $+0.0023 \pm 0.028 \mathrm{I}$ & 0.0608 \\
\hline
\end{tabular}

Notes: Data are presented as mean $\pm \mathrm{SD}$. $\Delta$ means the difference between the value at baseline and the value after 6 months. Abbreviations are as in Table I. the change of adiponectin was $+6.43 \pm 4.89 \mathrm{ng} / \mathrm{mL}$ in the pioglitazone group and $-0.09 \pm 3.04 \mathrm{ng} / \mathrm{mL}$ in the glimepiride group, representing a significant increase in the pioglitazone group $(P<0.0001)$. The LDL-Rm ratio, which reflects LDL particle size, was decreased in the pioglitazone group and increased in the glimepiride group. These results indicate that LDL particle size in the pioglitazone group was increased. However, the difference between the two groups was not significant ( $P=0.0608$, Table 2 ). Other parameters were not significantly different between the two groups (Table 2). No drug-related adverse effects were observed in any of the patients.

Pioglitazone significantly decreased the mean (standard error) CAVI from $9.59 \pm 0.16$ to $9.25 \pm 0.17(P<0.001)$ after 6 months (Figure 1A). Mean (standard error) CAVI increased slightly, but not significantly, in the glimepiride group (from $9.32 \pm 0.26$ to $9.42 \pm 0.27$; Figure $1 \mathrm{~B}$ ). The changes in CAVI during this study are shown in Figure $1 \mathrm{C}$. The mean (standard error) decrease in CAVI was significantly greater in the pioglitazone group than in the glimepiride group $(-0.337 \pm 0.088$ versus $0.107 \pm 0.151, P<0.05)$.

The correlation between change in CAVI and changes in various parameters was analyzed in all subjects to clarify the important factors involved in the decrease in CAVI. Among the factors analyzed, changes in body weight, body mass index, adiponectin level, and LDL-Rm ratio had a significant correlation with a decrease in CAVI (Table 3). The changes in systolic and diastolic BP were not correlated with a change in CAVI. The change in fasting blood glucose was significantly different between the two groups, but was not correlated with the change in CAVI.

\section{Discussion}

In the present study, pioglitazone as add-on therapy significantly increased body weight and body mass index and improved fasting blood glucose and serum adiponectin compared with glimepiride as add-on therapy in patients with type 2 diabetes receiving metformin. Only pioglitazone add-on therapy decreased CAVI significantly, whereas glimepiride add-on therapy did not decrease CAVI. The decrease in CAVI correlated with the increase in body weight, body mass index, serum adiponectin, and LDL-Rm ratio. There were no correlations between the change in CAVI and changes in systolic and diastolic BP.

Cardiovascular disease is a major cause of death in patients with type 2 diabetes. ${ }^{1}$ Metformin, an insulin sensitizer, reduces the incidence of myocardial infarction compared with sulfonylureas. ${ }^{5}$ This finding suggests that 
A

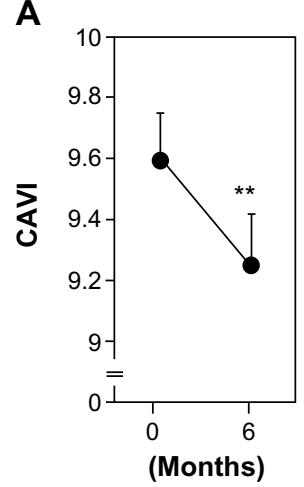

B

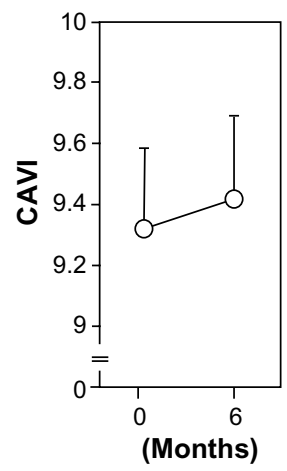

C

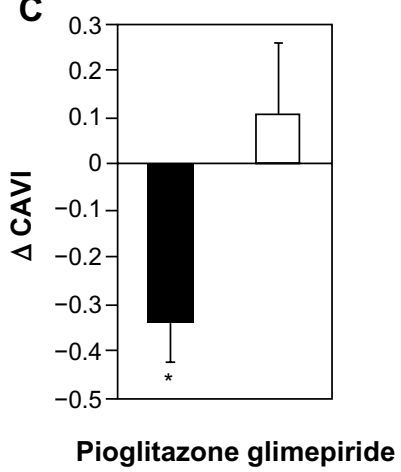

Figure I (A) Changes in cardio-ankle vascular index (CAVI) before and after 6 months of pioglitazone therapy. Data are presented as the mean \pm standard error of the mean $* * P<0.01$ versus 0 month, paired $t$-test. (B) Changes in CAVI before and after 6 months of glimepiride therapy. Data are presented as the mean \pm standard error of the mean. (C) Changes in CAVI after 6 months of pioglitazone or glimepiride therapy. The closed bar denotes the pioglitazone group and the open bar denotes the glimepiride group. Data are presented as the mean \pm standard error of the mean. $\Delta$ indicates the difference between the value at baseline and the value after 6 months. $* P<0.05$ versus glimepiride group, unpaired $t$-test.

it is important to reduce insulin resistance in patients with type 2 diabetes mellitus. However, when metformin monotherapy cannot control blood glucose, should the next step of diabetes treatment be to increase insulin secretion or to reduce insulin resistance? In the present study, pioglitazone as add-on therapy improved CAVI, a surrogate marker of cardiovascular disease, in patients with type 2 diabetes who were already receiving metformin. Glimepiride as add-on therapy did not decrease CAVI in patients with type 2 diabetes on metformin. The change in fasting blood glucose was significantly different between pioglitazone and glimepiride, but was not correlated with the change in CAVI. Adiponectin, a well known marker of insulin resistance, was increased only

Table 3 Correlation between change in CAVI and changes in various parameters after 6 months in all subjects

\begin{tabular}{lll}
\hline & $\begin{array}{l}\text { Correlation } \\
\text { coefficient }(\boldsymbol{r})\end{array}$ & P value \\
\hline$\Delta \mathrm{BW}$ & -0.2993 & 0.0202 \\
$\Delta \mathrm{BMI}$ & -0.2993 & 0.0202 \\
$\Delta \mathrm{SBP}$ & 0.2299 & 0.0773 \\
$\Delta \mathrm{DBP}$ & 0.1754 & 0.1800 \\
$\Delta \mathrm{FBG}$ & $-0.023 \mathrm{I}$ & 0.8608 \\
$\Delta \mathrm{HbA} \mathrm{I}_{\mathrm{lc}}$ & -0.0980 & 0.4562 \\
$\Delta \mathrm{TC}$ & 0.0710 & 0.5899 \\
$\Delta \mathrm{TG}$ & 0.0542 & 0.6807 \\
$\Delta \mathrm{HDL}-\mathrm{C}$ & -0.1828 & 0.1622 \\
$\Delta \mathrm{LDL}-\mathrm{C}$ & -0.2074 & 0.1118 \\
$\Delta$ preheparin LPL mass & $-0.076 \mathrm{I}$ & $0.563 \mathrm{I}$ \\
$\Delta$ adiponectin & -0.3503 & $0.006 \mathrm{I}$ \\
$\Delta$ MDA-LDL & 0.0896 & 0.4958 \\
$\Delta$ urinary albumin & -0.2052 & 0.1158 \\
$\Delta$ LDL-Rm ratio & 0.4053 & 0.0013 \\
\hline Notes: Corr & &
\end{tabular}

Notes: Correlation coefficient was calculated for change in CAVI ratio vs, change in each parameter. $\Delta$ means the difference between the value at baseline and the value after 6 months. Abbreviations are as in Table I. in the pioglitazone group. The change in adiponectin between the pioglitazone and glimepiride groups was significantly different, and was correlated with the change in CAVI. Thus, our results suggest that reducing insulin resistance is more important than increasing insulin secretion in patients with type 2 diabetes not adequately controlled on metformin. Other clinical studies support our results. ${ }^{26,27}$

Although arterial stiffness is usually evaluated by baPWV, baPWV is correlated with BP. ${ }^{17}$ In a previous study, pioglitazone improved baPWV but also decreased systolic BP. ${ }^{14}$ Therefore, it is uncertain if pioglitazone improves arterial stiffness in diabetic patients, and we considered it necessary to evaluate the effect of pioglitazone on arterial stiffness with CAVI. We found that pioglitazone decreased systolic and diastolic BP. However, glimepiride also decreased systolic and diastolic BP, and these changes were not significant between the pioglitazone and glimepiride groups. Further, the change in CAVI was not correlated with changes in systolic or diastolic BP. Thus, we confirmed that pioglitazone improves arterial stiffness in type 2 diabetic patients.

Serum adiponectin reflects insulin resistance and is also related to atherosclerosis and cardiovascular disease. Serum adiponectin levels have an inverse correlation with the risk of high carotid intima-media thickness. ${ }^{28}$ Further, there has been a clinical study showing that adiponectin has a strong association with aortic stiffness in patients after acute STsegment elevation myocardial infarction ${ }^{29}$ and that serum adiponectin levels were increased more by pioglitazone than glimepiride. Thus, we conclude that serum adiponectin is an important factor in the mechanism via which pioglitazone improved CAVI in this study.

The LDL-Rm ratio, which reflects LDL particle size, correlated significantly with CAVI in patients with attenuated 
CAVI after 6 months in our present study. The LDL-Rm ratio was decreased in the pioglitazone group and increased in the glimepiride group. Small-sized LDL is a known risk factor for coronary heart disease. ${ }^{30}$ Troglitazone, a TZD, has been observed to increase LDL particle size in a previous study. ${ }^{13}$ We consider that enlargement of LDL particle size might be an important reason for the decrease in CAVI seen in the current study, although comparison of the change in LDL-Rm ratio between the two groups indicates that there was no significant difference.

Body weight and body mass index were significantly increased in the pioglitazone group compared with the glimepiride group, and these changes were correlated with the decrease in CAVI. CAVI was not correlated with body mass index in a previous study. ${ }^{31}$ Pioglitazone is well known for increasing body weight. Only pioglitazone significantly decreased CAVI in our study. We consider the effects of pioglitazone to be the reason for the correlation between body weight or body mass index and CAVI.

Glimepiride decreased CAVI in type 2 diabetic patients not treated with metformin, and this attenuating effect on CAVI was due to improved insulin resistance. ${ }^{32}$ In the present study, however, glimepiride add-on therapy did not increase adiponectin, a well known marker of insulin resistance. ${ }^{8} \mathrm{We}$ speculate that glimepiride is incapable of reducing insulin resistance in patients with type 2 diabetes who were already treated with metformin and, consequently, could not decrease CAVI in this study.

The main limitation of this study is its short duration of only 6 months. Thus, the long-term effect of pioglitazone and prognosis are still unclear. Further investigation with longer-term evaluations might be necessary. However, we were able to demonstrate an effect of pioglitazone on arterial stiffness in this study.

\section{Conclusion}

Pioglitazone add-on therapy in patients with type 2 diabetes already treated with metformin achieved improvement in CAVI, a BP-independent arterial stiffness parameter and a surrogate marker of cardiovascular disease. These effects were not observed with glimepiride add-on therapy. These results suggest that pioglitazone added on to metformin improves arterial stiffness independent of BP.

\section{Author contributions}

Masahiro Ohira worked on substantial contributions to conception and design, acquisition of data, or analysis and interpretation of data. Takashi Yamaguchi worked on acquisition of data. Atsuhito Saiki worked on acquisition of data. Noriko Ban worked on acquisition of data. Hidetoshi Kawana worked on acquisition of data. Ayako Nagumo worked on acquisition of data. Takeyoshi Murano worked on analysis and interpretation of data. Kohji Shirai worked on interpretation of data. Ichiro Tatsuno worked on analysis and interpretation of data. All authors worked on revising the article critically for important intellectual content, final approval of the version to be published and agreement to be accountable for all aspects of the work in ensuring that questions related to the accuracy or integrity of any part of the work are appropriately investigated and resolved.

\section{Disclosure}

The authors report no conflicts of interest in this work.

\section{References}

1. Lee WL, Cheung AM, Cape D, Zinman B. Impact of diabetes on coronary artery disease in women and men: a meta-analysis of prospective studies. Diabetes Care. 2000;23:962-968.

2. Stratton IM, Adler AI, Neil HA, et al. Association of glycaemia with macrovascular and microvascular complications of type 2 diabetes (UKPDS 35): prospective observational study. BMJ. 2000;321: 405-412.

3. [No authors listed]. UK Prospective Diabetes Study (UKPDS) Group. Intensive blood-glucose control with sulphonylureas or insulin compared with conventional treatment and risk of complications in patients with type 2 diabetes (UKPDS 33). Lancet. 1998;352:837-853.

4. Stettler C, Allemann S, Jüni P, et al. Glycemic control and macrovascular disease in type 1 and 2 diabetes mellitus: meta-analysis of randomized trials. Am Heart J. 2006;152:27-38.

5. [No authors listed]. UK Prospective Diabetes Study (UKPDS) Group. Effect of intensive blood-glucose control with metformin on complication in over weight patients with type 2 diabetes (UKPDS 34). Lancet. 1998;352:854-865.

6. Scarpello JH, Howlett HC. Metformin therapy and clinical uses. Diab Vasc Dis Res. 2008;5:157-167.

7. Koenig W, Karakas M, Zierer A, et al. Oxidized LDL and the Risk of Coronary Heart Disease: Results from the MONICA/KORA Augsburg Study. Clin Chem. 2011;57:1196-1200.

8. Weyer C, Funahashi T, Tanaka S, et al. Hypoadiponectinemia in obesity and type 2 diabetes: close association with insulin resistance and hyperinsulinemia. J Clin Endocrinol Metab. 2001;86:1930-1935.

9. Saiki A, Oyama T, Endo K, et al. Preheparin serum lipoprotein mass might be a biomarker of metabolic syndrome. Diabetes Res Clin Pract. 2007;76:93-101.

10. Staels B, Fruchart JC. Therapeutic roles of peroxisome proliferatoractivated receptor aonist. Diabetes. 2005;54:2460-2470.

11. Shakuto S, Oshima K, Tsuchiya E. Glimepiride exhibits prophylactic effect on atherosclerosis in cholesterol-fed rabbits. Atherosclerosis. 2005;182:209-217.

12. Subramanian V, Golledge J, Ijaz T, Bruemmer D, Daugherty A. Pioglitazone-induced reductions in atherosclerosis occur via smooth muscle cell-specific interaction with PPAR \{gamma . Circ Res. 2010;107:953-958.

13. Shirai K, Itoh Y, Sasaki H, et al. The effect of insulin sensitizer, troglitazone, on lipoprotein lipase mass in preheparin serum. Diabetes Res Clin Pract. 1999;46:35-41.

14. Kato T, Sawai Y, Kanayama H, et al. Comparative study of low-dose pioglitazone or metformin treatment in Japanese diabetic patients with metabolic syndrome. Exp Clin Endocrinol Diabetes. 2009;117: 593-599. 
15. Shirai K, Utino J, Otsuka K, Takata M. A novel blood pressureindependent arterial wall stiffness parameter; cardio-ankle vascular index (CAVI). J Atherosclerosis Thromb. 2006;13:101-107.

16. Takaki A, Ogawa H, Wakayama T, et al. Cardio-ankle vascular index is a new noninvasive parameter of arterial stiffness. Circ J. 2007;71: 1710-1714.

17. Shirai K, Song M, Suzuki J, et al. Contradictory effects of $\beta 1$ - and $\alpha 1$-aderenergic receptor blockers on cardio-ankle vascular stiffness index (CAVI)- CAVI independent of blood pressure. J Atheroscler Thromb. 2011;18:49-55.

18. Kubozono T, Miyata M, Ueyama K, et al. Clinical significance and reproducibility of new arterial distensibility index. Circ J. 2007;71: 89-94.

19. Nakamura K, Tomaru T, Yamamura S, Miyashita Y, Shirai K, Noike H. Cardio-ankle vascular index is a candidate predictor of coronary atherosclerosis. Circ J. 2008;72:598-604.

20. Shirai K, Nema T, Hiroh Y, Itoh Y, Miyashita Y, Watanabe H. Clinical efficacy of the direct assay method using polymers for serum high density lipoprotein cholesterols. J Clin Lab Anal. 1997;11: 82-86.

21. Kobayashi J, Hashimoto H, Fukamachi I, et al. Lipoprotein lipase mass and activity in severe hypertriglyceridemia. Clin Chim Acta. 1993;216: 113-123.

22. Ohira M, Endo K, Oyama T, et al. Improvement of postprandial hyperglycemia and arterial stiffness upon switching from premixed human inuslin 30/70 to biphasic insulin aspart 30/70. Metabolism. 2011;60: 78-85.

23. Kotani K, Maekawa M, Kanno T, Kondo A, Toda N, Manabe M. Distribution of immunoreactive malondialdehyde-modified low-density lipoprotein in human serum. Biochim Biophys Acta. 1994;1215: $121-125$.
24. Mead MG, Dangerfield WG. The investigation of midband lipoproteins using polyacrylamide gel electrophoresis. Clin Chim Acta. 1974;51: 173-182.

25. Miwa K. Low density lipoprotein particles are small in patients with coronary vasospasm. Int J Cardiol. 2003;87:193-201.

26. Mazzone T, Meyer PM, Feinstein SB, et al. Effect of pioglitazone compared with glimepiride on carotid intima-media thickness in type 2 diabetes: a randomized trial. JAMA. 2006;296:2572-2581.

27. Nissen SE, Nicholls SJ, Wolski K, et al; PERISCOPE investigators. Comparison of pioglitazone vs glimepiride on progression of coronary atherosclerosis in patients with type 2 diabetes: the PERISCOPE randomized controlled trial. JAMA. 2008;299:1561-1573.

28. Yoon JH, Kim SK, Choi HJ, et al. Adiponectin provides additional information to conventional cardiovascular risk factors for assessing the risk of atherosclerosis in both genders. PLoS One. 2013;8:e75535.

29. Reinstadler S, Klug G, Feistritzer H, et al. Relation of plasma adiponectin levels and aortic stiffness after acute ST-segment elevation myocardial infarction. Eur Heart J Acute Caridiovasc Care. 2014;3:10-17.

30. Austin MA, Breslow JA, Hennekens CH, Buring JE, Willett WC, Krauss RM. Low density lipoprotein subclass pattern and risk of myocardial infarction. J Am Med Assoc. 1988;260:1917-1921.

31. Nagayama D, Endo K, Ohira M, et al. Effects of body weight reduction on carido-ankle vascular index (CAVI). Obes Res Clin Pract. 2013;7:e89-e164.

32. Nagayama D, Saiki A, Endo K, et al. Improvement of cardio-ankle vascular index by glimepiride in type 2 diabetic patients. Int J Clin Pract. 2010;64:1796-1801.

\section{Publish your work in this journal}

Diabetes, Metabolic Syndrome and Obesity: Targets and Therapy is an international, peer-reviewed open-access journal committed to the rapid publication of the latest laboratory and clinical findings in the fields of diabetes, metabolic syndrome and obesity research. Original research, review, case reports, hypothesis formation, expert opinion and commentaries are all considered for publication. The manuscript management system is completely online and includes a very quick and fair peer-review system, which is all easy to use. Visit http://www.dovepress.com/testimonials.php to read real quotes from published authors. 